\title{
GENERATING FUNCTIONS OF THE PRODUCTS OF BIVARIATE COMPLEX FIBONACCI POLYNOMIALS WITH GAUSSIAN NUMBERS AND POLYNOMIALS
}

\author{
Souhila Boughaba, Ali Boussayoud ${ }^{1}$
}

AND

NABIHA SABA

LMAM Laboratory and Department of Mathematics Mohamed Seddik Ben Yahia University, Jijel, Algeria

e-mail: souhilaboughaba@gmail.com

aboussayoud@yahoo.fr

sabarnhf1994@gmail.com

\begin{abstract}
In this paper, we define and study the bivariate complex Fibonacci and Lucas polynomials. We introduce a operator in order to derive some new symmetric properties of bivariate complex Fibonacci and bivariate complex Lucas polynomials, and give the generating functions of the products of bivariate complex Fibonacci polynomials with Gaussian Fibonacci, Gaussian Lucas and Gaussian Jacobsthal numbers, Gaussian Pell numbers, Gaussian Pell Lucas numbers. By making use of the operator defined in this paper, we give some new generating functions of the products of bivariate complex Fibonacci polynomials with Gaussian Jacobsthal, Gaussian Jacobsthal Lucas polynomials and Gaussian Pell polynomials.
\end{abstract}

Keywords: symmetric functions, generating functions, bivariate complex Fibonacci polynomials, bivariate complex Lucas polynomials.

2010 Mathematics Subject Classification: Primary 05E05; Secondary $11 \mathrm{~B} 39$.

\section{REFERENCES}

[1] A. Abderrezzak, Généralisation de la transformation d'Euler d'une série formelle, Adv. Math. 103 (1994) 180-195. doi:10.1006/aima.1994.1008

\footnotetext{
${ }^{1}$ Corresponding author.
} 
[2] M. Asci and E. Gurel, On Bivariate Complex Fibonacci and Lucas Polynomials, Conference on Mathematical Sciences ICM 2012, March 11-14, 2012.

[3] M. Asci and E. Gurel, Gaussian Jacobsthal and Gaussian Jacobsthal Lucas polynomials, Notes Number Theory Discrete Math. 19 (2013) 25-36.

[4] C. Bolat and H. Kose, On the properties of k-Fibonacci numbers, Int. J. Contemp. Math. Sci. 5 (2010) 1097-1105.

[5] S. Boughaba, A. Boussayoud, S. Araci and M. Kerada, Construction of Generating Functions of Gaussian Fibonacci Numbers and Polynomials, Univ. J. Math. Appl. (Submitted).

[6] S. Boughaba and A. Boussayoud, On some identities and generating function of Both K-jacobsthal numbers and symmetric functions in several variables, Konuralp J. Math. 7 (2019) 235-242.

[7] S. Boughaba and A. Boussayoud, Construction of symmetric functions of generalized Fibonacci numbers, Tamap J. Math. Stat. 3 (2019) 1-8. doi:10.29371/2019.16.SI01

[8] S. Boughaba, A. Boussayoud and Kh. Boubellouta, Generating functions of modified Pell numbers and bivariate complex Fibonacci polynomials, Turkish J. Anal. Number 7 (2019) 113-116. doi:10.12691/tjant-7-4-3

[9] A. Boussayoud, On some identities and generating functions for Pell-Lucas numbers, Online J. Anal. Comb. 12 (2017) 1-10.

[10] A. Boussayoud, L'action de L'opérateur $\delta_{e_{1} e_{2}}^{k}$ sur la Série $\sum_{n=0}^{\infty} S_{n}(A) e_{1}^{n} z^{n}$, Doctoral dissertation (Mohamed Seddik Ben Yahia University, Jijel, Algeria, 2017).

[11] A. Boussayoud and N. Harrouche, Complete symmetric functions and k-Fibonacci numbers, Commun. Appl. Anal. 20 (2016) 457-465.

doi:10.12732/caa.v20i4.1

[12] A. Boussayoud, M. Kerada and M. Boulyer, A simple and accurate method for determination of some generalized sequence of numbers, Int. J. Pure Appl. Math. 108 (2016) 503-511. doi:10.12732/ijpam.v108i3.3

[13] A. Boussayoud, M. Kerada and R. Sahali, Symmetrizing operations on some orthogonal polynomails, Int. Electron. J. Pure Appl. Math. 9 (2015) 191-199. doi:10.12732/iejpam.v9i3.8

[14] A. Boussayoud and M. Kerada, Symmetric and generating functions, Int. Electron. J. Pure Appl. Math. 7 (2014) 195-203. doi:10.12732/iejpam.v7i4.5

[15] A. Boussayoud, M. Kerada, R. Sahali and W. Rouibah, Some applications on generating functions, J. Concr. Appl. Math. 12 (2014) 321-330.

[16] A. Boussayoud and R. Sahali, The application of the operator $L_{b_{1} b_{2}}^{-k}$ in the series $\sum_{j=0}^{+\infty} a_{j} b_{1}^{j} z^{j}$, J. Adv. Res. Appl. Math. 7 (2015) 68-75. 
[17] G.B. Djordjevic, Generating functions of the incomplete generalized Fibonacci and generalized Lucas numbers, Fibonacci Q. 42 (2004) 106-113.

[18] G.B. Djordjevic and H.M. Srivastava, Incomplete generalized Jacobsthal and Jacobsthal-Lucas numbers, Math. Comput. Modelling 42 (2005) 1049-1056. doi:10.1016/j.mcm.2004.10.026

[19] S. Falcon and A. Plaza, On the Fibonacci k-numbers, Chaos Solitons Fractals 32 (2007) 1615-1624. doi:10.1016/j.chaos.2006.09.022

[20] S. Falcon and A. Plaza, k-Fibonacci sequences and polynomials and their derivatives, Chaos Solitons Fractals 39 (2009) 1005-1019.

doi:10.1016/j.chaos.2007.03.007

[21] S. Falcon and A. Plaza, The k-Fibonacci sequence and the Pascal 2-triangle, Chaos Solitons Fractals 33 (2008) 38-49. doi:10.1016/j.chaos.2006.10.022

[22] D. Foata and G.-N. Han, Nombres de Fibonacci et polynômes orthogonaux, Leonardo Fibonacci : il tempo, le opere, l'eredità scientifica (1994) 179-200.

[23] S. Halici and S. Oz, On some Gaussian Pell and Pell-Lucas numbers, Ordu Univ. J. Sci. Tech. 6 (2016) 8-18.

[24] S. Halici and S. Oz, On Gaussian Pell polynomials and their some properties, Palest. J. Math. 7 (2018) 251-256.

[25] A.F. Horadam, Generating functions for powers of a certain generalized sequence of numbers, Duke Math. J. 32 (1965) 437-446. doi:10.1215/S0012-7094-65-03244-8

[26] A.F. Horadam and E.M. Horadam, Roots of recurrence-generated polynomials, Fibonacci Quart. 20 (1982) 219-226.

[27] A.F. Horadam and J. M. Mahon, Pell and Pell-Lucas polynomials, Fibonacci Quarterly 23 (1985) 7-20.

[28] J.H. Jordan, Gaussian Fibonacci and Lucas numbers, Fibonacci Quarterly 3 (1965) $315-318$.

[29] D.V. Kruchinin and V.V. Kruchinin, Application of a composition of generating functions for obtaining explicit formulas of polynomials, J. Math. Anal. Appl. 404 (2013) 161-171. doi:10.1016/j.jmaa.2013.03.009

[30] I.G. Macdonald, Symmetric Functions and Hall Polynomias (Oxford University Press, Oxford, 1979)

[31] I. Mezo, Several generating functions for second-order recurrence sequences, J. Integer Seq. 12 (2009) 1-16.

[32] A. Pintér and H.M. Srivastava, Generating functions of the incomplete Fibonacci and Lucas numbers, Rend. Circ. Mat. Palermo 48 (1999) 591-596. doi:10.1007/BF02844348 
[33] D. Tasci and M. Cetin Firengiz, Incomplete Fibonacci and Lucas p-numbers, Math. Comput. Modelling 52 (2010) 1763-1770.

doi:10.1016/j.mcm.2010.07.003

Received 29 December 2019

Revised 22 February 2020

Accepted 3 July 2020 